\title{
Commentary \\ Modulating sensitivity to drug-induced apoptosis: the future for chemotherapy?
}

\author{
Guy Makin ${ }^{\star \dagger}$ and Caroline Dive*
}

${ }^{*}$ CRC Molecular and Cellular Pharmacology Group, School of Biological Sciences, Manchester, UK

${ }^{+}$Medical School, University of Manchester, Manchester, UK

Correspondence: Guy Makin, CRC Molecular and Cellular Pharmacology Group, School of Biological Sciences, G38 Stopford Building, Oxford Road, Manchester M13 9PT, UK. E-mail: guy.makin@man.ac.uk

Received: 28 February 2001

Breast Cancer Res 2001, 3:150-153

Revisions requested: 7 March 2001

Revisions received: 8 March 2001

Accepted: 13 March 2001

(C) 2001 BioMed Central Ltd

Published: 28 March 2001

(Print ISSN 1465-5411; Online ISSN 1465-542X)

\begin{abstract}
Drug resistance is a fundamental problem in the treatment of most common human cancers. Our understanding of the cellular mechanisms underlying death and survival has allowed the development of rational approaches to overcoming drug resistance. The mitogen activated protein kinase family of protein serine/threonine kinases has been implicated in this complex web of signalling, with some members acting to enhance death and other members to prevent it. A recent publication by MacKeigan et al is the first to demonstrate an enhancement of drug-induced cell death by simultaneous blockade of MEK-mediated survival signalling, and offers the potential for targeted adjuvant therapy as a means of overcoming drug resistance.
\end{abstract}

Keywords: apoptosis, drug resistance, MEK, paclitaxel

Improvements in chemotherapy for some malignancies, such as childhood leukaemia, have resulted in considerable increases in survival. However, many of the more common adult cancers, including carcinoma of the breast, remain stubbornly resistant to drug treatment, despite dose escalation and the increasing use of stem cell support [1]. The explosion of interest in apoptosis in the past 10 years has been underpinned by the hope that a greater understanding of the way in which cancer cells die after chemotherapy-induced damage would allow the development of a more rational approach to overcoming the problem of drug resistance. Unless cells receive external survival signals, they will 'default' to apoptosis [2]. These signals are provided by soluble cytokines and growth factors, cell-extracellular matrix contact and cell-cell contact. The overall survival threshold is probably determined by the balance of interactions between members of the Bcl-2 family of proteins on the cytoplasmic surface of internal membranes, such as the outer mitochondrial membrane. These pro-apoptotic or antiapoptotic proteins can homodimerise or heterodimerise, and a satisfying but still unproven hypothesis is that, by doing so, they either activate or neutralise each other depending on the balance of death and survival stimuli.

A wide range of chemotherapeutic agents is able to trigger apoptosis (reviewed in [3]). In this model of their action, chemotherapeutic drugs drive cell death by generating damage signals at their locus of action (eg DNA damage), and these signals become integrated at Bcl-2 family protein containing complexes where the decision to undergo apoptosis is taken and signalled to apoptosis effector molecules. This is classical stimulus response coupling, and it is the efficiency of this coupling that determines the threshold for

ERK = extracellular signal-related kinase; IL = interleukin; JNK = c-Jun $\mathrm{N}$-terminal kinase; MAP = mitogen activated protein; MEK = MAP kinase kinase; $M E K K=$ MEK kinase; MKK = MAP kinase kinase. 
survival. A complex of proteins, describing this coupling and activation centre, has been conceptualised as an 'apoptosome', and contains the precursors of the proteolytic enzymes (caspases) that cleave key cellular proteins to generate the apoptotic morphology [4]. While many mechanisms of drug resistance have been defined in which drug-target interactions are modified, it is also important to consider the impact of the cellular environment on the coupling of drug-induced damage to the activation of the 'apoptosome', via modification of Bcl-2 family proteins.

Cell survival in vivo depends on ligation of surface receptors by soluble factors and both cell-cell and cell-matrix interactions. The signalling pathways activated by these receptors ultimately impinge upon Bcl-2 family members, and this may be a key mechanism mediating drug resistance. Such a survival signalling pathway exists between interleukin (IL-3) receptor ligation and the pro-apoptotic protein $\mathrm{Bad}$ [5]. It is thus possible that signals from growth factors and cytokines provide not only mitogenic cues, but also discrete survival signals that raise the survival threshold of tumours and contribute to drug resistance. Loss of IL-3 in pro-B lymphocytes leads to the upregulation of the pro-apoptotic Bcl-2 family protein Bim, via the Forkhead transcription factor FKHL-1, and this induces apoptosis [6]. In a B-cell lymphoma model, the provision of extrinsic survival signals attenuates etoposide-induced exposure of the $\mathrm{N}$-terminus of Bax, an early step in the activation of this proapoptotic protein [7]. In the mouse mammary epithelial cell model, loss of cell-substrate contact-mediated ligation of integrin receptors results in a conformational change in the $\mathrm{N}$-terminus of Bax, and its subsequent translocation to the mitochondria [8]. These conformational changes in Bax, and its subsequent translocation to mitochondria, are mediated by p38 mitogen activated protein (MAP) kinase in nitric oxide induced apoptosis in neurons [9]. A further link between protein serine/threonine kinase signalling and this part of the cell death response is provided by the recent observation that c-Jun N-terminal kinase (JNK) is involved in the coupling of DNA damage to mitochondrial cytochrome $c$ release in fibroblasts [10].

This brings us neatly from the general concepts of apoptosis and cell survival to the specific roles of the extracellular signal-related kinase (ERK)/MAP kinase family of protein kinases in the regulation of cell death. This family of kinases consists of proline-directed serine/threonine kinases that are activated by dual phosphorylation on tyrosine and threonine, and that are widespread among living organisms. In mammals, they exist mainly in cascades containing three kinases functioning in series. In broad terms, there are three distinct families. In the ERK1/ERK2 module, growth factor derived extracellular signals are translated to Raf-1 activation, which leads to the phosphorylation of MAP kinase kinase (MEK) 1 and MEK2; these in turn phosphorylate and activate ERK1 and ERK2. In the stress-activated protein kinase/JNK module, a wide range of stimuli including UV light and osmotic shock result in activation of MEK kinase (MEKK)1, and then MAP kinase kinase (MKK) 4 and MKK7, which phosphorylate JNK. The final member of the family is p38 MAP kinase, which is also activated by stress and inflammatory cytokines such as tumour necrosis factor- $\alpha$ and IL-1, MEKK1 and MKK3 and MKK4 (for a review, see [11]). Although these pathways share many similarities, they are clearly independent: MEK1/MEK2 do not phosphorylate JNK or p38, and MKK3/MKK4/MKK7 do not phosphorylate ERK1/ERK2 [12]. A widely accepted model is that the balance between growth factor activated ERK and stress-activated JNK and p38 pathways determines whether the cell lives or dies. In the rat phaeochromcytoma cell line PC-12, withdrawal of nerve growth factor leads to sustained JNK and p38 MAP kinase activity, inhibition of ERK activity, and apoptosis, which can be prevented by transfection of constitutively active MEK1 mutants [13]. Hippocampal neurons in jnk 3 knockout mice do not undergo excitoxininduced apoptosis [14], and JNK is needed for apoptosis of immature T lymphocytes in developing mice [15]. JNK is also needed for UV-induced apoptosis in mouse fibroblasts, which intriguingly is mediated via a failure of mitochondrial cytochrome $c$ release, suggesting further interaction between the Bcl-2 family and the MAP kinase signalling cascades [10]. Further subtlety is lent to this system by the discovery that, while MEKK $1^{-/-}$embryonic stem cell lines lose their JNK response to microtubule disruption and cold shock, this kinase is not needed for JNK activation by UV irradiation or heat shock [16]. Furthermore, this loss of MEKK1-mediated activation of JNK leads to an increased apoptotic response to hyperosmolarity and microtubule disruption, suggesting that the survival or death specificity of these pathways may depend upon the precise nature of the signal inducing them. JNK does not appear to be needed for Fas-mediated or tumour necrosis factor-mediated apoptosis [17] and, in some situations, may protect cells from apoptosis.

The discovery of small molecule inhibitors of the MAP kinase pathways facilitated the transition from the theoretical framework already described to the implementation of novel therapies. The first inhibitor to be identified was PD 098059, which inhibits MEK without affecting JNK or p38, and is able to prevent call growth and reverse the phenotype of ras-transformed cells [18]. U0126 is a further non-competitive MEK1/MEK2 inhibitor, which is also specific for these kinases. The major advantage of U0126 over PD 098059 is its 100-fold greater affinity for MEK, thus making it more suited to in vivo applications [19]. The efficacy of this approach has been demonstrated in vivo with an even more effective MEK inhibitor, PD 184352. This compound is able to completely inhibit MEK activity in a range of human tumour cell lines at concentrations as low as $100 \mathrm{nM}$, without effecting JNK or 
p38 activity. This compound was active in vivo against xenografts from a range of tumour cell lines, its activity correlating with the expression levels of MEK within the cell lines [20]. This overexpression of MEK within experimentally induced tumours has also been demonstrated in vivo in certain types of brain tumours and, pertinently, in breast carcinoma [21]. Thus, not only is the theoretical basis for the use of MEK inhibition to modulate survival signalling in place but effective drugs are available, and the target is overexpressed in breast cancer. But with which chemotherapeutic drugs should these inhibitors be used?

The alkaloid drug paclitaxel (Taxol) is a relatively novel anticancer agent. Paclitaxel has a wide range of activities in vivo against relatively drug-resistant solid tumours and a unique mode of action. Unlike the Vinca alkaloids, paclitaxel binds preferentially to polymerised tubulin and shifts the dynamic balance between tubulin dimers and microtubules towards microtubule assembly. Paclitaxel ultimately causes mitotic block at the metaphase/anaphase boundary by the suppression of dynamic instability (the transition between phases of lengthening and shortening) at the ends of mitotic spindle microtubules [22]. Paclitaxel treatment of lymphoblasts in culture results in a rapid increase in JNK activity and a reduction in ERK2 activity [23]. Bcl-2 can also be phosphorylated by JNK [24], providing a further link between paclitaxel, MAP kinase cascades and Bcl-2 family proteins. The in vivo significance of these observations remains to be clarified.

MacKeigan et al [25] have used the theoretical framework discussed in this paper to augment the pro-apoptotic activity of paclitaxel in breast, lung and ovarian carcinoma cell lines. They show that treatment with nanomolar concentrations of paclitaxel results in increases in JNK and ERK1/ERK2 activity, and that the latter is specifically blocked by micromolar concentrations of the MEK inhibitor U0126. Paclitaxel-induced apoptosis is significantly increased by U0126, PD98059, or by dominantnegative MEK, and this effect is considerably more than additive. This logical approach to the modulation of druginduced apoptosis is exactly what was hoped would arise from an understanding of cell death, illustrating well the principle that apoptosis results from either loss of survival signals (provided in this setting by ERKs) or death signals (in the present study from JNK). The power of the approach of MacKeigan et al [25] is partly that it tilts both sides of the balance, both activating death signals and inhibiting survival signals, but that they are also able to demonstrate that clinically relevant concentrations of drug can be made dramatically more potent with readily available agents. With the in vivo efficacy of small molecule MAP kinase inhibitors already demonstrated, their use to enhance the efficacy of chemotherapeutic agents in clinical trials is a realistic goal. Perhaps the future of chemotherapy is no longer so bleak.

\section{References}

1. Budman DR, Berry DA, Cirrincione CT, Henderson IC, Wood WC, Weiss RB, Ferree R, Muss HB, Green MR, Norton L, Frei E: Dose and dose intensity as determinants of outcome in the adjuvant treatment of breast cancer. J Natl Cancer Inst 1998, 90:1205-1211.

2. Raff MC: Social controls on cell survival and cell death. Nature 1993, 356:397-400.

3. Hickman JA, Dive C: Apoptosis and Cancer Chemotherapy. Totowa, NJ: Humana Press, 1999.

4. Chinnaiyan AM: The apoptosome: heart and soul of the cell death machine. Neoplasia 1999, 1:5-15.

5. Zha JP, Harada H, Yang E, Jockel J, Korsmeyer SJ: Serine phosphorylation of death agonist Bad in response to survival factor results in binding to 14-3-3 not Bcl-X(L). Cell 1996, 87: 619-628.

6. Dijkers PF, Medema RH, Lammers J-WJ, Koenderman L, Coffer $\mathrm{PJ}$ : Expression of the pro-apoptotic $\mathrm{Bcl}-2$ family member Bim is regulated by the forkhead transcription factor FKHR-L1. Curr Biol 2000, 10:1201-1204.

7. Taylor ST, Hickman JA, Dive C: Epigenetic determinants of resistance to etoposide regulation of $\mathrm{Bcl}-\mathrm{x}(\mathrm{L})$ and $\mathrm{Bax}$ by tumor microenvironmental factors. J Natl Cancer Inst 2000, 92:18-23.

8. Gilmore AP, Metcalfe AD, Romer $\mathrm{LH}$, Streuli $\mathrm{CH}$ : Integrin-mediated survival signals regulate the apoptotic function of Bax through its conformation and subcellular localization. J Cell Biol 2000, 149:431-445.

9. Ghatan S, Larner S, Kinoshita Y, Hetman M, Patel L, Xia Z, Youle RJ, Morrison RS: p38 MAP kinase mediates Bax translocation in nitric oxide-induced apoptosis in neurons. J Cell Biol 2000, 150:335-347.

10. Tournier C, Hess $P$, Yang DD, Xu J, Turner TK, Nimnual A, BarSagi D, Jones SN, Flavell RA, Davis RJ: Requirement of JNK for stress-induced activation of the cytochrome c-mediated death pathway. Science 2000, 288:870-874.

11. English J, Pearson G, Wilsbacher J, Swantek J, Karandikar M, Xu $\mathrm{S}$, Cobb MH: New insights into the control of MAP kinase pathways. Exp Cell Res 1999, 253:255-270.

12. Derijard B, Raingeaud J, Barrett T, Wu I-H, Han J, Ulevitch RJ, Davis RJ: Independant human MAP kinase signal transduction pathways defined by MEK and MKK isoforms. Science 1995, 267:682-685.

13. Xia Z, Dickens M, Raingeaud J, Davis RJ, Greenberg ME: Opposing effects of ERK and JNK-p38 MAP kinases on apoptosis. Science 1995, 270:1326-1331.

14. Yang DD, Kuan CY, Whitmarsh AJ, Rincon M, Zheng TS, Davis RJ, Rakic P, Flavell RA: Absence of excitotoxicity-induced apoptosis in the hippocampus of mice lacking the Jnk3 gene. Nature 1997, 389:865-870.

15. Rincon M, Whitmarsh A, Yang DD, Weiss L, Derijard B, Jayaraj $P$, Davis RJ, Flavell RA: The JNK pathway regulates the in vivo deletion of immature CD4 ${ }^{+} \mathrm{CD}^{+}{ }^{+}$thymocytes. J Exp Med 1998, 188:1817-1830.

16. Yujiri T, Sather S, Fanger GR, Johnson GL: Role of MEKK1 in cell survival and activation of JNK and ERK pathways defined by targeted gene disruption. Science 1998, 282:1911-1914.

17. Natoli G, Costanzo A, lanni A, Templeton DJ, Woodgett JR, Balsano C, Levrero M: Activation of SAPK/JNK by TNF receptor 1 through a noncytotoxic TRAF2-dependent pathway. Science 1997, 275:200-203.

18. Dudley DT, Pang L, Decker SJ, Bridges AJ, Saltiel AR: A synthetic inhibitor of the mitogen activated protein kinase cascade. Proc Natl Acad Sci USA 1995, 92:7686-7689.

19. Favata MF, Horiuchi KY, Manos EJ, Daulerio AJ, Stradley DA, Feeser WS, Van Dyk DE, Pitts WJ, Earl RA, Hobbs F, Copeland RA, Magolda RL, Scherle PA, Trzaskos JM: Identification of a novel inhibitor of mitogen-activated protein kinase kinase. $J$ Biol Chem 1998, 273:18623-18632.

20. Sebolt-Leopold JS, Dudley DT, Herrera R, Becelare KV, Wiland A, Gowan RC, Tecle H, Barrett SD, Bridges A, Przybranowski S, Leopold WR, Saltiel AR: Blockade of the MAP kinase pathway suppresses growth of colon tumours in vivo. Nat Med 1999, 5: 810-816.

21. Sivaraman VS, Wang H, Nuovo GJ, Malbon CC: Hyperexpression of mitogen activated protein kinase in human breast tissue. J Clin Invest 1997, 99:1478-1483. 
22. Jordan MA, Toso RJ, Thrower D, Wilson L: Mechanism of mitotic block and inhibition of cell proliferation by taxol at low concentrations. Proc Natl Acad Sci USA 1993, 90:9552-9556.

23. Amato SF, Swart JM, Berg M, Wanebo HJ, Mehta SR, Chiles TC: Transient stimulation of the c-Jun- $\mathrm{NH}_{2}$-terminal kinase/activator protein 1 pathway and inhibition of extracellular signalrelated kinase are early events in paclitaxel-mediated apoptosis in human B lymphoblasts. Cancer Res 1998, 58: 241-247.

24. Maundrell $K$, Antonsson B, Magnenat E, Camps M, Muda M, Chabert C, Gillieron C, Boschert U, Vial-Knecht E, Martinou J-C, Arkinstall S: Bcl-2 undergoes phosphorylation by c-Jun $\mathrm{N}$-terminal kinase/stress-activated protein kinases in the presence of the constitutively active GTP-binding protein Rac1. J Biol Chem 1997, 272:25238-25242.

25. MacKeigan JP, Collins TS, Ting JP-Y: MEK inhibition enhances paclitaxel-induced tumor apoptosis. J Biol Chem 2000, 275: 38953-38956. 